\title{
Bridgehead double bonds
}

\author{
Josef Michl,* Juliusz G. Radziszewski, John W. Downing, Jan Kopecky, Piotr Kaszynski \\ Department of Chemistry, University of Utah, Salt Lake City, UT 84112, U.S.A. \\ and \\ Robert D. Miller \\ IBM Almaden Research Center, 650 Harry Road, K91/801, San Jose, CA 95120, U.S.A.
}

Abstract - A survey is given of recent progress in our laboratory in the preparation and spectroscopic characterization of compounds containing highly twisted bridgehead $\mathrm{C}=\mathrm{N}$ and $\mathrm{C}=\mathrm{C}$ double bonds.

\section{INTRODUCTION}

This survey is an update on an earlier one ${ }^{1}$ and represents a progress report on the preparation and spectroscopy of strongly twisted $\mathrm{C}=\mathrm{N}$ and $\mathrm{C}=\mathrm{C}$ double bonds. Our interest in these structures was awakened in the course of investigations of photochemical mechanisms, in which biradicaloids ${ }^{2}$ and biradicaloid geometries of ordinary molecules ${ }^{3}$ play a crucial role.

\section{THEORY}

In our work, the twist angle $\theta$ in a bridgehead imine has been defined as the dihedral angle between the planes defined by the $\mathrm{C}=\mathrm{N}$ bond and (i) the $\mathrm{N}-\mathrm{C}$ bond and (ii) an axis passing through the unsaturated bridgehead carbon so as to form equal angles with the three bridgehead bonds. In a similar fashion, for a bridgehead alkene, the definition of $\theta$ has been the dihedral angle between the planes defined by the $C=C$ bond and ( 1 ) an axis passing through the bridgehead unsaturated carbon so as to form equal angles with the three bonds originating in this carbon and (ii) a similarly defined axis passing through the other unsaturated carbon. Both cases are illustrated on examples in Fig. 1. Although these are perhaps not the most common definitions, we have found them useful and meaningful in that the axes in question approximate the axes of the p orbitals available for perturbed $\pi$ bonding. Recently, the label POAV1 was proposed for this usage and an 


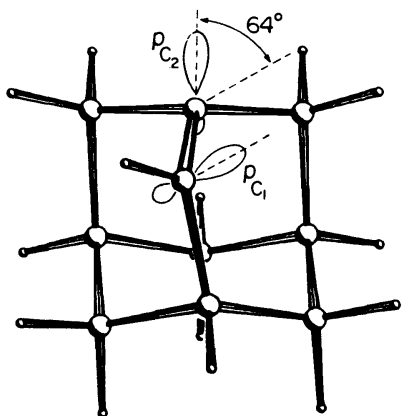

15

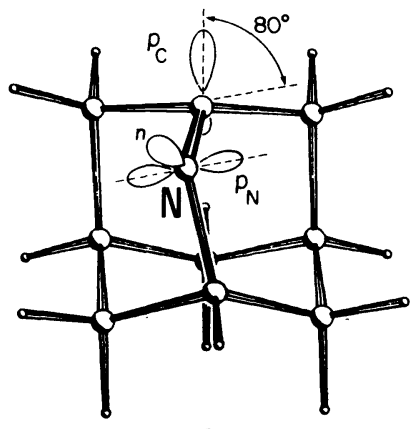

10

Fig. 1 MNDO equilibrium geometries of adamantene (left) and 2-azaadamant-1-ene (right). The orbital axes used in the definition of the twist angle $\theta$ and the orbitals used in the description of $\pi$ bonding are indicated.
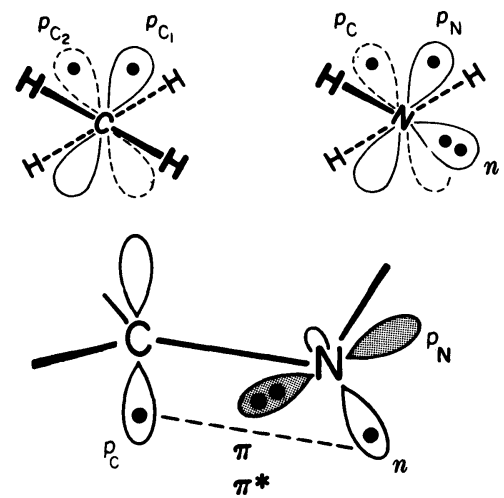

Fig. 2 Top, a Newman projection of the primary orbitals involved in the description of twisted $\mathrm{C}=\mathrm{C}$ and $\mathrm{C}=\mathrm{N}$ double bonds. Bottom, a perspective view of these orbitals in an orthogonally twisted $\mathrm{C}=\mathrm{N}$ bond.

extensive discussion was published. ${ }^{4}$ The definitions imply that the twist angle $\theta$ in a bridgehead trisubstituted alkene (but not imine) can be varied within a wide range merely by moving the vinylic hydrogen so as to pyramidalize the $=\mathrm{CH}-$ carbon to various degrees, without moving any of the heavier atoms. The presence of such pyramidalization in the ground state of bridgehead alkenes was predicted a long time ago ${ }^{5}$ and if the theory is correct, it is clear that unlike an imine, a trisubstituted alkene cannot be forced into an orthogonal twist, $\theta=90^{\circ}$, by incorporation into a bridgehead position.

Other geometrical parameters of potential importance in the description of bonding in bridgehead imines and alkenes are the valence angle $\phi$ on the $N$ or $=\mathrm{CH}$ - atoms, the pyramidalization angle $\omega$ on the unsaturated bridgehead carbon atom, and in alkenes also the pyramidalization angle $\omega^{\prime}$ on the other unsaturated carbon atom. The pyramidalization angle on an atom is defined by the amount by which the angle between the axis of its $\pi$-bonding hybrid orbital (Fig. 1), and any one of the three bonds this carbon carries, exceeds $90^{\circ}$. Although within the range of compounds investigated in our work, the variation of the calculated angle $\omega$ is not negligible and ranges up to the $\mathrm{sp}^{3}$ (tetrahedral) value of $19.5^{\circ}$, we believe that the twist angle $\theta$, and in the imines also the valence angle $\phi$, and in the alkenes also the pyramidalization angle $\omega^{\prime}$, are of primary interest. 
Alkenes. The behavior of the $\pi$ and $\pi^{*}$ orbitals and of the ground $S_{0}$ $\left(\pi^{2}\right)$ and excited $S_{1}$ singlet $\left(\pi^{*}\right)$ states of the $C=C$ bond as a function of $\theta$, with a large barrier in $S_{0}$ and a minimum in $S_{1}$ at orthogonality, are well known and need not be described in detail. We shall only point out that the pyramidalization at the unsaturated bridgehead carbon undoubtedly contributes to the weakening of the $\mathrm{C}=\mathrm{C}$ double bond. For instance, for $\mathrm{a}$ bond calculated to be pyramidalized to $\omega=14^{\circ}$ on each terminus, the observed $C=C$ stretching frequency is lowered from 1685 to $1557 \mathrm{~cm}^{-1} 6$

Imines. The electronic states involved in the twisting of $\mathrm{C}=\mathrm{N}$ double bonds are intrinsically complicated by the intimate interplay between the $\mathrm{p}_{C}$ and $p_{N}$ orbitals of the planar $\pi$ system and the approximately $s^{2}$ hybridized lone pair orbital $(n)$ on the nitrogen atom (Figs. 1,2). 1,7,8 At the planar geometry $\left(\theta=0^{\circ}\right)$, the $n$ orbital does not interact with $\mathrm{P}_{\mathrm{C}}$ nor with $\mathrm{P}_{\mathrm{N}}$, whose mutual interaction is strong. At orthogonal twist $\left(\theta=90^{\circ}, \mathrm{Fig} .2\right)$, the interaction between $p_{C}$ and $p_{N}$ vanishes, and that between $p_{C}$ and $n$ is maximized. The lone pair is then housed in the $\mathrm{p}_{\mathrm{N}}$ orbital. At intermediate twist angles $\theta, p_{C}$ interacts with $p_{N}$ and with $n$ as well, but $p_{N}$ and $n$ still do not interact. At these geometries, the $\mathrm{C}=\mathrm{N}$ bond is isoelectronic with the allyl anion. The strength of the $\mathrm{p}_{C}-\mathrm{n}$ interaction is a function of the degree to which $n$ has $p$ character and therefore depends on the valence angle $\phi$ on the nitrogen atom. It is maximized at $\phi=180^{\circ}$, in which case the twist angle $\theta$ is obviously immaterial. Variation of either one of the angles $\theta$ and $\phi$ alone can bring about a syn-anti interconversion, and an infinite number of intermediate paths are also imaginable.

The $\mathrm{p}_{\mathrm{C}}, \mathrm{p}_{\mathrm{N}}$, and $\mathrm{n}$ orbitals of the allyl-anion-like $\mathrm{C}=\mathrm{N}$ system interact to yield three combinations that can be called $\pi, n$, and $\pi^{*}$ at planar geometries $\left(\theta=0^{\circ}\right)$; we keep these labels for convenience even for twisted geometries. Twisting at a constant valence angle $\phi$ does not change the energies of the $\pi$ and $\pi^{*}$ orbitals much, but it greatly increases the energy of the $n$ orbital which gradually loses all of its partial s character and acquires pure $p$ character at $\theta=90^{\circ}$, as noted above. 
The effect of the twisting at constant $\phi$ on state energies is related simply to its effect on orbital energies. The configuration of the ground state, $S_{0}$, is $\pi^{2} n^{2}$ and its energy increases slowly as $\theta$ goes from $0^{\circ}$ to $90^{\circ}$ (Fig. 3). This results in a large barrier along the twisting syn-anti isomerization path, at least for the usual values of the valence angle $\phi$, near $110^{\circ}$. This barrier can be viewed as due in part to the increase in the energy of the lone pair, which loses all of its partial $s$ character, and in part to the replacement of the efficient $p_{C}-p_{N} \pi$ interaction at $\theta=0^{\circ}$ by the less effective $p_{C}-n$ interaction at $90^{\circ}$. As the valence angle $\phi$ increases and approaches $180^{\circ}$, this loss becomes less severe, since the $n$ orbital begins to look more like a pure $p$ orbital; at $\phi=180^{\circ}$ the barrier obviously disappears. As a result, the lowest-energy path for thermal synanti isomerization involves no twisting but a mere valence angle change. Along this path, the only penalty paid is the increase in the energy of the $n$ orbital as it goes from $s p^{2}$ to purely $p$ in character, and the $\pi$ bond remains intact. Note, however, that also at the more usual values of $\phi$, the $\mathrm{C}=\mathrm{N}$ bond remains double even at orthogonality: the $\mathrm{p}_{\mathrm{C}}$ orbital can always find a suitable partner for a perturbed $\pi$ interaction by choosing an optimal combination of the $\mathrm{p}_{\mathrm{N}}$ and $\mathrm{n}$ orbitals, and at orthogonality this choice is pure n (Fig. 2). Except for the case $\phi=90^{\circ}$, in which $n$ has essentially only s character, a $\pi$ bond results, even though it may be weak. 1

The configuration of the lowest excited singlet state $S_{1}$ is $n \pi^{*}$ and its energy drops as $\theta$ goes from $0^{\circ}$ to $90^{\circ}$ (Fig. 4). Its minimum occurs at $\theta=$ $90^{\circ}, \phi \simeq 110^{\circ}$, in the vicinity of a geometry where the $S_{0}$ and $S_{1}$ surfaces touch, providing a funnel for the return of excited singlet molecules to a region of the ground state from which relaxation to either the syn or the anti equilibrium geometry will occur. The origin of the $\mathrm{S}_{0}-\mathrm{S}_{1}$ touching can be understood easily in terms of a simple correlation diagram. An analysis is available elsewhere ${ }^{8}$ and shows that the barrier in $S_{0}$ and the minimum in $S_{1}$ at $\theta=90^{\circ}$ twist can be viewed as a result of a crossing which is avoided for most choices of the valence angle $\phi$. Inspection of Figs. 3 and 4 shows that the $S_{0}$ and $S_{1}$ surfaces are set up ideally for an efficient photochemical syn-anti isomerization involving a twisting motion. 


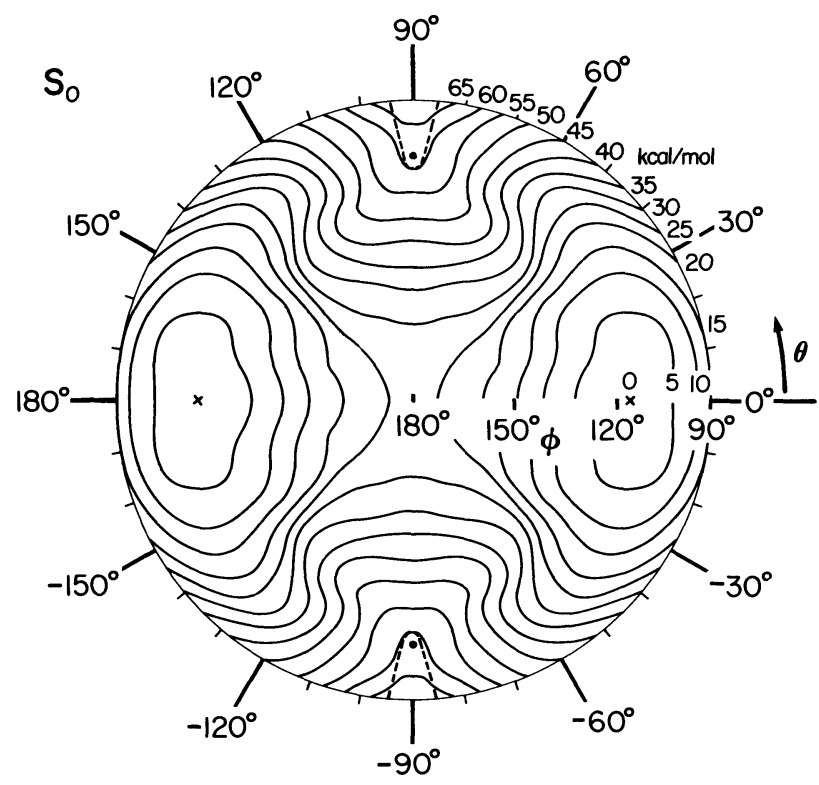

Fig. 3 An energy contour map for the $S_{0}$ state of formaldimine. The radial variable $\phi$ and the angular variable $\theta$ are defined in the text. Energies in $\mathrm{kcal} / \mathrm{mol}$ are given relative to that at the two equilibrium geometries (syn and anti), indicated by crosses at $\theta=$ $0^{\circ}$ and $180^{\circ}$, and $\phi=115^{\circ}$. The dots at $\theta= \pm 90^{\circ}, \phi=106.5^{\circ}$ represent the $S_{0}-S_{1}$ touching points. The dashed lines indicate the $S_{0}-T_{1}$ intersection. Calculated by an ab initio MRD-CI procedure. Reproduced by permission from ref. 8, where further details are given.

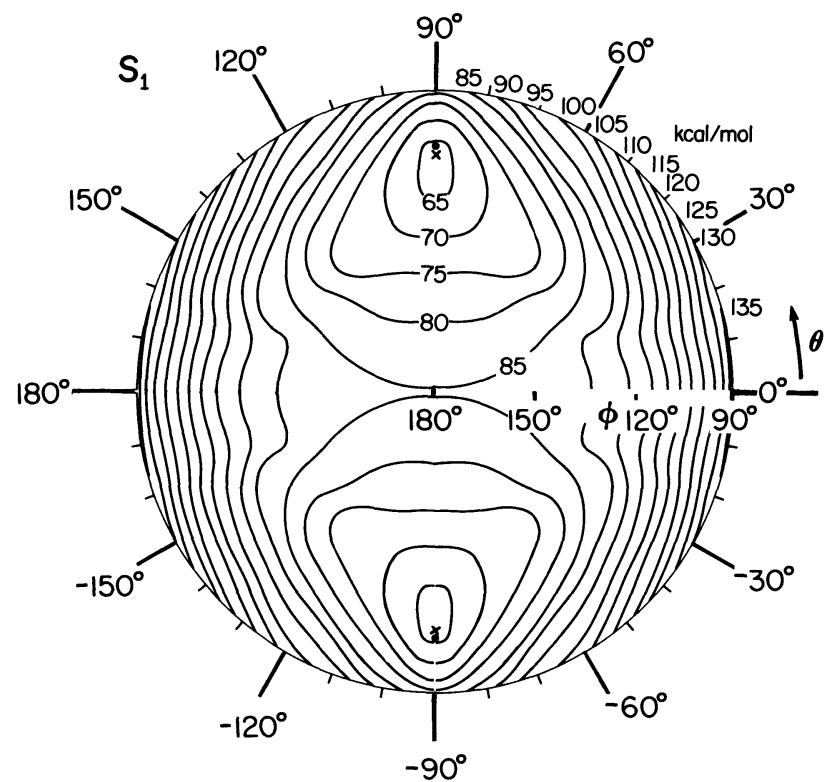

Fig. 4. An energy contour map for the $S_{1}$ state of formaldimine; see caption to Fig. 3. The crosses at $\theta= \pm 90^{\circ}, \phi=109^{\circ}$ represent the positions of the minima in $S_{1}$. Reproduced by permission from ref. 8, where further details are given. 
The configuration of the second excited singlet state is $\pi \pi^{*}$ and its energy should not depend much on the twist angle $\theta$. Of course, since the ground state energy rises rapidly with an increasing twist, the $\pi \pi^{*}$ excitation energy should still drop, but less rapidly than the $n \pi^{*}$ excitation energy.

\section{TWISTED $C=N$ DOUBLE BONDS}

We have prepared a series of bridgehead azides, deposited them in argon matrix, and converted them to photoproducts by UV irradiation. The primary products are $\mathrm{N}_{2}$, a trace of a bridgehead nitrene, and a bridgehead imine or imines.

Scheme 1

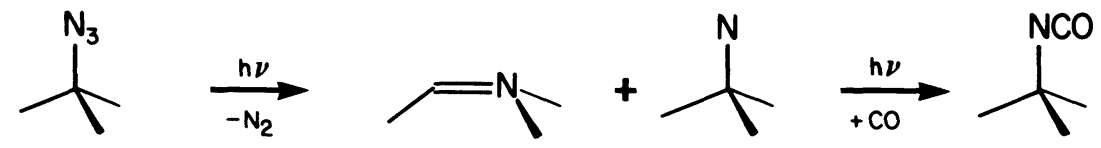

Molecular nitrogen was detected by Raman spectroscopy. The nitrenes were readily detected by EPR and UV spectroscopy but their concentration was not sufficient to obtain IR spectra. Only after photochemical addition of CO doped into the matrix was it possible to detect the $-\mathrm{N}=\mathrm{C}=0$ stretching vibration of the resulting isocyanate. After matrix warm-up, the isocyanate was identified by gas chromatography - mass spectroscopy.

The structures of the bridgehead imines produced were secured by chemical trapping (either addition of methanol or dimerization to yield products that were compared with authentic samples) and by UV-visible, IR and Raman spectroscopy on ordinary and ${ }^{15} \mathrm{~N}$-labeled samples. The imines are ESR-inactive. They are all sensitive to further irradiation at least to some degree, and their selective photodestruction with monochromatic light of suitable wavelengths was used to separate the spectra of components in mixtures of several imines by computer subtraction. Selective bleaching in the presence of trapping agents followed by warm-up and identification of the trapping products permitted us to attribute structures to the observed spectra. The measured and computed spectral characteristics of the imines are collected in Table 1; most of these results have already been published. ${ }^{1,7-10}$ 
Table 1

Properties of Bridgehead Imines

Calculated $^{\mathrm{a}}$ Ceometry $^{\mathrm{b}}$

$$
\text { (deg) }
$$

$\begin{array}{rrrr}\text { Imine } & \theta & \phi & \omega \\ 1 & 0 & 126 & 0 \\ 2 & 39 & 114 & 13 \\ 3 & 48 & 117 & 9 \\ 4 & 51 & 115 & 11 \\ 5 & 52 & 118 & 8 \\ 6 & 54 & 102 & 17 \\ 7 & 61 & 108 & 18 \\ 8 & 69 & 108 & 12 \\ 9 & 74 & 112 & 13 \\ 10 & 77 & 110 & 12 \\ 11 & 84 & 96 & 23\end{array}$

$\mathrm{C}=\mathrm{N}$ Stretching Frequency

$$
\left(\mathrm{cm}^{-1}\right)
$$

exp. calc. ${ }^{d}$

$1669 \quad(1669)^{\mathrm{f}}$

1586

1597

1591

1600

- 1489

- 1530

1480

1475

1451

-

1396 $n \pi *$ Excitation Energy $\left(10^{3} \mathrm{~cm}^{-1}\right)$

exp. calc.

41.7

30.0

35.3

28.9

34.8

27.2

31.9

26.6

33.1

26.3

24.7

(32.5)

23.6

24.8

20.3

25.0

19.5

20.2

17.4

\begin{tabular}{ll}
$-\quad 15.3$ \\
\hline
\end{tabular} $\pi \pi^{*}$ Excitation Energy

$$
\left(10^{3} \mathrm{~cm}^{-1}\right)
$$

exp. ${ }^{c}$ calc.

$\checkmark 55 \quad 56.4$

$49.5 \quad 43.9$

$48.5 \quad 44.6$

$49.0 \quad 43.6$

$46.7 \quad 44.2$

\begin{tabular}{l}
$-\quad 35.0$ \\
\hline
\end{tabular}

\begin{tabular}{l}
$-\quad 36.4$ \\
\hline
\end{tabular}

$42.2 \quad 35.4$

- $\quad 37.9$

$39.4 \quad 38.2$

29.9

$a_{M N D O ;} b_{\theta:}$ Double bond twist angle, $\phi: N$ or $=C H-$ valence angle, $w$ : bridgehead carbon pyramidalization angle;

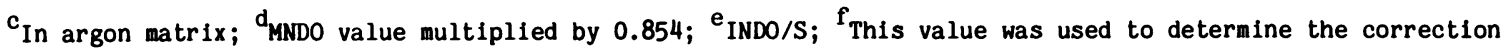
factor, 0.854 .

\section{Scheme 2}

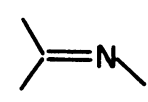

I

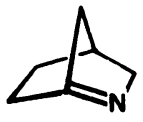

6

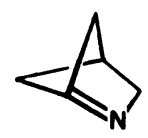

II

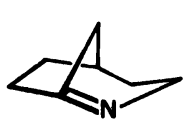

2

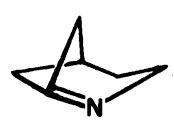

7

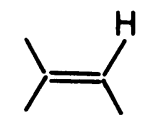

12

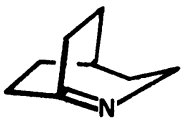

3

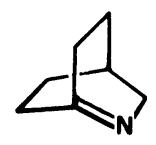

8

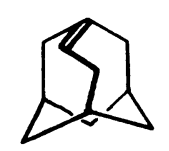

13

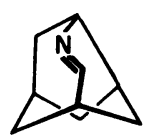

4

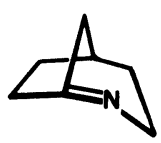

9

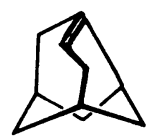

14

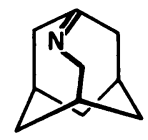

5

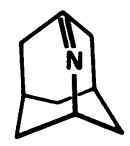

10

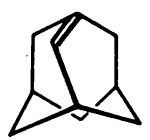

15

Syn-anti isomerism at the bridgehead was observed only in the case of the $Z$ (2) and $E(9)$ isomers of 2-azatricyclo[3.2.1]oct-1-ene. The latter is very easily photoisomerized to the former. The one observed isomer of 4azaprotoadamant-3-ene has therefore been assigned the more stable structure 4, which is also supported by its IR and UV-visible spectra. In the more symmetrical imines such as 2-azahomoadamant-1-ene 5, the $\mathrm{Z}$ and $\mathrm{E}$ isomers 
represent enantiomers of each other. In the case of 5, a partial photoresolution with circularly polarized light has been accomplished. ${ }^{7}$

Vibrational spectra. The $\mathrm{C}=\mathrm{N}$ stretching frequencies of the bridgehead imines (Table 1), identified by ${ }^{15} \mathrm{~N}$ labeling, are substantially lower than that of acetone methylimine (1), which can be viewed as a standard planar trialkylated imine. In imines containing the $\mathrm{C}=\mathrm{N}$ bond in a transazacycloheptene ring, the shift is about $100 \mathrm{~cm}^{-1}$, and in those containing a trans-azacyclohexene $r$ ing, it is about $200 \mathrm{~cm}^{-1}$. Within the range or compounds studied, the $\mathrm{C}=\mathrm{N}$ stretching frequency correlates well with the cosine of the twisting angle $\theta$ at the MNDO equilibrium geometry (Fig. 5). This would be expected if the valence angles $\phi$ and pyramidalization angles $\omega$ in all of these imines were roughly comparable. The MNDO geometries (Table 1) indeed suggest that this is moderately well fulfilled. The correlations of the stretching frequencies with $\phi$ and $w$ are not nearly as good as the correlation with $\cos \theta$.

The $\mathrm{C}=\mathrm{N}$ stretching frequencies calculated by the MNDO method also exhibit an approximate linear dependence on the computed value of $\cos \theta$, and when they are multiplied by the correction factor 0.854 , which brings the value calculated for 1 into agreement with the experimental value, they all also agree very nicely with the observed values (Fig. 5). The surprisingly good agreement gives us confidence that the computed twist angles $\theta$ cannot be completely spurious. Judging by the $\mathrm{C}=\mathrm{N}$ stretching frequencies, 2-azadamant-1-ene (10) is the most highly twisted bridgehead imine prepared so far, and the MNDO value for its twist angle is nearly 80 degrees.

Linear extrapolation to $\theta=90^{\circ}$ twist may be somewhat risky. Still, it suggests that the $\mathrm{C}=\mathrm{N}$ stretching frequency of a truly orthogonal imine with a valence angle $\phi$ comparable to those computed for our imines $\left(95-115^{\circ}\right)$ will be about $1350-1400 \mathrm{~cm}^{-1}$, roughly $300 \mathrm{~cm}^{-1}$ below the values usual for planar trialkylimines. This is still considerably higher than the values typical of the stretching frequency of single $\mathrm{C}-\mathrm{N}$ bonds, even those involving a $\mathrm{sp}^{2}$ hybridized carbon atom. Most of the difference is probably due to the fact that even an orthogonally twisted $C=N$ bond remains double because the $p_{C}-n$ interaction is still present as discussed above (Fig. 2), but some may be 


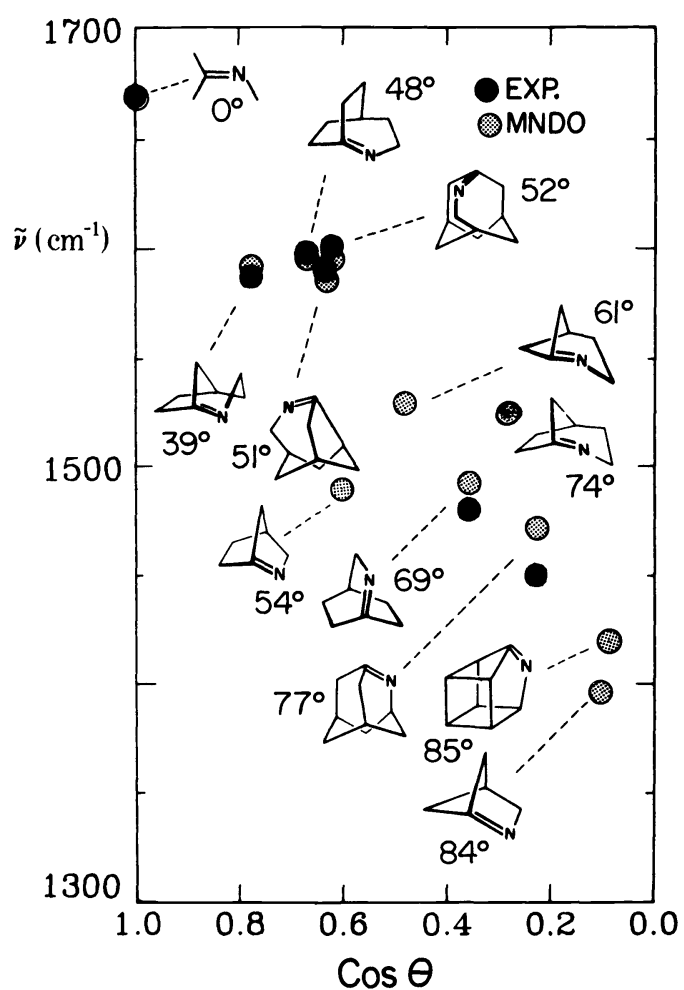

Fig. 5. Bridgehead imines: $C=N$ stretching frequencies plotted against calculated $\cos \theta$. Dark points: experimental; light points: calculated (MNDO). The calculated twist angles are shown.

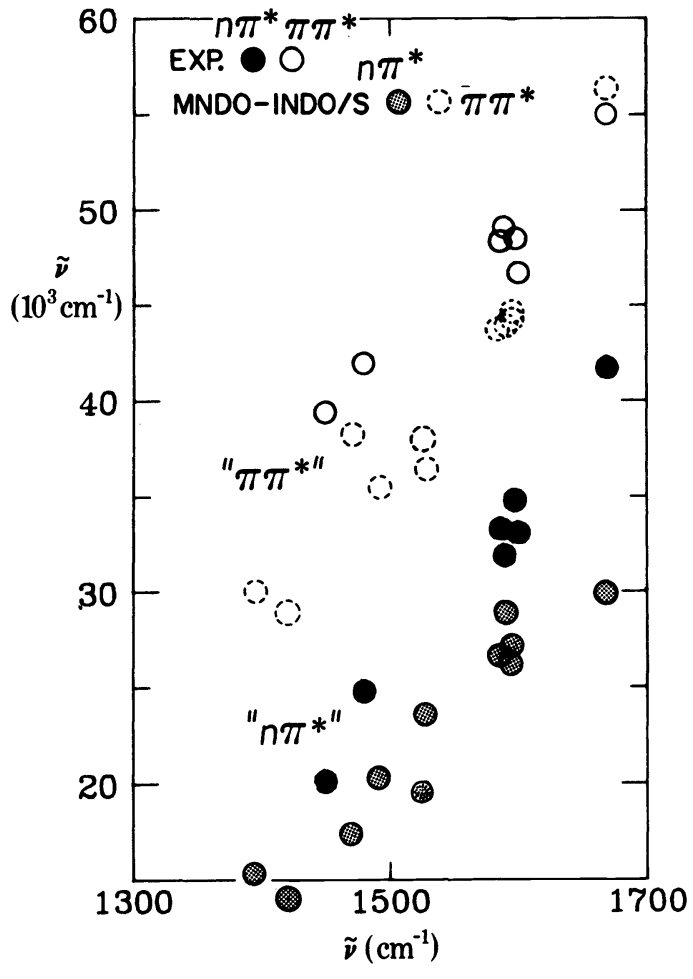

Fig. 6. Bridgehead imines: electronic excitation energies plotted against the $\mathrm{C}=\mathrm{N}$ stretching frequency. Empty circles: $\pi \pi^{*}$, experimental; empty dashed circles: $\pi \pi^{*}$, calculated; black circles: $n \pi^{*}$, experimental; gray circles: $n \pi^{*}$, calculated. Calculations by INDO/S at MNDOoptimized geometries.

due to a changed coupling of the $C=N$ stretching motion to other skeletal motions in the observed normal mode. The increased importance of such coupling in the more highly strained bridgehead imines is revealed by the ${ }^{15} \mathrm{~N}$ isotopic shifts in their IR spectra. While in the less twisted imines only the $C=N$ stretching vibration shifts considerably $\left(s 20 \mathrm{~cm}^{-1}\right)$, in the more highly twisted ones a large number of IR bands shift by significant amounts. Regardless of the detailed nature of the relation between the $C=N$ stretching frequency and the $\mathrm{C}=\mathrm{N}$ stretching force constant, Fig. 5 suggests strongly that the value of the $\mathrm{C}=\mathrm{N}$ frequency can be used as a measure of the degree of twist.

Electronic spectra. The $n \pi^{*}$ and $\pi^{*}$ excitation energies of the bridgehead imines are also quite unusual (Table 1) and are considerably redshifted relative to ordinary planar trialkylated imines. On an absolute energy scale, the shifts are somewhat larger for the $n \pi^{*}$ transition, as expected from theory. Fig. 6 shows that both the $n \pi^{*}$ and the $\pi \pi^{*}$ excitation 
energies are correlated with the $\mathrm{C}=\mathrm{N}$ stretching frequency and thus with the calculated $\cos \theta$. The magnitude of the $n \pi^{*}$ shifts is impressive: in transazacycloheptene rings, about $10,000 \mathrm{~cm}^{-1}$, and in trans-azacyclohexene rings, about $20,000 \mathrm{~cm}^{-1}$; the $n^{*}$ excitation energy of the red 2-azaadamant-1-ene (10) is nearly $3 \mathrm{eV}$ lower than that of the colorless strain-free 1. For an exactly orthogonal imine with a valence angle comparable to those in our imines, linear extrapolation yields an expected $n \pi^{*}$ excitation energy of the order of $10-15,000 \mathrm{~cm}^{-1}$, corresponding to the edge of the IR region, and an expected $\pi \pi^{*}$ excitation energy of about $30-35,000 \mathrm{~cm}^{-1}$.

The $n \pi^{*}$ and $\pi \pi^{*}$ excitation energies calculated by the INDO/S method at the MNDO-optimized ground state geometries are also show in Fig. 6. They are all about $5000 \mathrm{~cm}^{-1}$ too low but describe the overall experimental trends very well and permit quite reasonable predictions for imines that have not yet been prepared.

Photoisomerization. The availability of a fair number of imines twisted to various degrees offers a rare opportunity to construct a cut through an $S_{1}$ potential energy surface along a photochemically relevant path relying largely on experimental data. If we ignore the variation in the angles $\phi$ and $\omega$ and adopt an MNDO calculation on 1 to approximate the variation of the $S_{0}$ energy with the twist angle $\theta$, we can use the experimental excitation energies to construct cuts through the $n \pi^{*}\left(S_{1}\right)$ and $\pi \pi^{*}\left(S_{2}\right)$ surfaces along the twisting path (Fig. 7 ). This yields a qualitative view of the surfaces involved in photochemical syn-anti isomerizations of imines, with the minimum through which the return of $S_{1}$ to $S_{0}$ presumably occurs clearly delineated at the orthogonal geometry. This qualitative picture is quite independent of the likely errors in the MNDO ground state energies. These energies cannot be calibrated to an experimental activation energy for the thermal syn-anti isomerization process since this most likely does not proceed by twisting and avoids the photochemical path displayed in Fig. 7 altogether. 


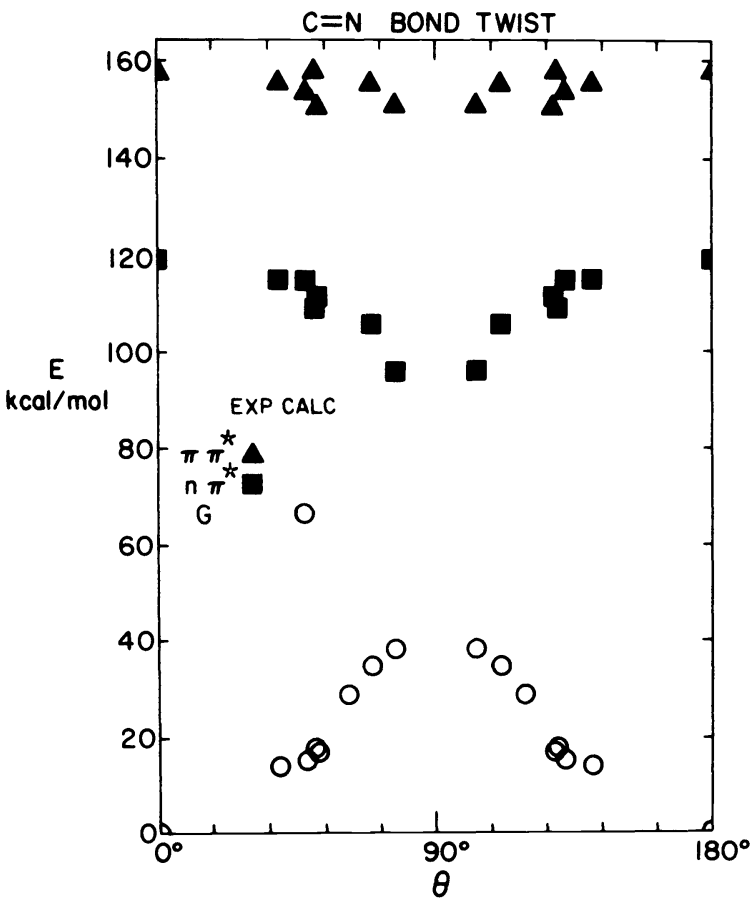

Fig. 7.

The MNDO $S_{0}$ surface for twisting 1 and the points for the $S_{1}\left(n \pi^{*}\right)$ and $S_{2}\left(\pi^{*}\right)$ surfaces obtained by adding observed excitation energies, as a function of the calculated twist angle $\theta$.

Table 2

Properties of Bridgehead Alkenes

Calculated Geometry ${ }^{a, b} \quad C=C$ Stretching Frequency

$$
\text { (deg) }
$$

Alkene $\quad \theta \quad \omega \quad \omega^{\prime}$

12000

$\begin{array}{llll}13 & 44 & 9 & 6\end{array}$

$14 \quad 44 \quad 11 \quad 6$

15

$$
\left(\mathrm{cm}^{-1}\right)
$$

exp. $^{c} \quad$ calcd. ${ }^{d}$

1675

$1610^{8}$

1587

1481 $\pi \pi^{*}$ Excitation Energy

$\begin{array}{cc}\text { exp. }^{c}{ }^{\mathrm{f}} & \text { calcd. } \\ & \left.544^{3} \mathrm{~cm}^{-1}\right) \\ - & 35.2 \\ s 40 & 34.7 \\ 31.5 & 24.6\end{array}$

$a_{M N D O ;} b_{\theta}$ : Double bond twist angle, $\omega$ : bridgehead pyramidalization angle, $\omega^{\prime}:$ methine pyramidalization angle; ${ }^{c}$ In argon matrix; ${ }^{d}$ MNDO value multiplied by 0.915 (a value chosen to optimize agreement for $C=C$ stretch in ethylene and tetramethylethylene); $\mathrm{e}_{\text {INDO/S; }} \mathrm{f}_{\text {In paraffin solution; }} \mathrm{g}_{\text {Measured on neat material }}{ }^{12}$

\section{TWISTED C $=$ C DOUBLE BONDS}

Less is known about the spectra of highly twisted bridgehead alkenes than bridgehead imines (Table 2). 1,11,12 Homoadamantene (13), analogous to 5 , has been prepared by pyrolysis of 1-adamantyldiazomethane. ${ }^{12}$ We have prepared adamantene (15), analogous to 10 , first by gas-phase dehalogenation of 1,2-dilodo and 1,2-dibromo derivatives of adamantane, ${ }^{11}$ and subsequently by matrix photolysis of 1-noradamantyldiazomethane. Our initial tentative 
identification of the $C=C$ stretching frequency in 15 was very uncertain ${ }^{1}$ since its peak in the IR spectrum is extremely weak. More recently, we have obtained a Raman spectrum of matrix-isolated 15 and we are now confident about the new value listed in Table 2. In addition to 15 , the irradiation of 1-noradamantyldiazomethane in argon matrix yields two other products, neither of which has been identified conclusively. By analogy to the structures obtained by the irradiation of azides, one or both might be the olefins expected from the shift of the "wrong" bond. The spectral properties of one of them indeed fit those anticipated for the more stable geometrical isomer of protoadamant-3-ene 14, analogous to 4 . Whether the other is the less stable isomer remains to be seen. All of the $C=C$ stretching frequency assignments listed in Table 2 are supported by deuteration shifts.

A comparison of Tables 1 and 2 suggests that the double bond stretching frequencies observed in a bridgehead imine and in the analogous alkene are quite close, being both dictated by the skeletal geometries. Once again, in a trans-cycloheptene $r$ ing the frequency is lowered by about $100 \mathrm{~cm}^{-1}$ and in a trans-cyclohexene ring by about $200 \mathrm{~cm}^{-1}$ relative to ordinary trialkylethylenes. This close similarity is found also with the MNDO calculated frequencies, which fit the observed values reasonably well after multiplication by a correction factor of $0.915^{6}$ which fits ethylene and tetramethylethylene to experiment. Indeed, the calculated equilibrium geometries in the imine and alkene series are very similar with respect to the location of the heavy atoms (Fig. 1). The large difference in the calculated twist angles $\theta$, about $15^{\circ}$ in the case of the pair 10-15, is virtually all due to the ability of the olefin to decrease $\theta$ by pyramidalizing its methine carbon, as discussed in the theoretical part above. The double bond frequencies listed in Table 2 suggest that the amount of bonding recovered in this fashion in a bridgehead olefin is comparable to that recovered by the intervention of the nitrogen lone pair in an analogous bridgehead imine. This result should facilitate the analysis of the vibrational spectra of new bridgehead olefins, which are generally harder to synthesize and characterize than the corresponding imines. 
Since the twist angle $\theta$ is always much lower in the bridgehead alkene than in its imine analog, the approximate equality of their double bond stretching frequencies implies that extrapolation to $\theta=90^{\circ}$, which gave 1350 - $1400 \mathrm{~cm}^{-1}$ for imines, will give a lower value for a truly orthogonal alkene. This is in keeping with the orthogonal imine still containing a double bond and the orthogonal alkene merely a single bond. We do not have enough data for bridgehead alkenes at present for a reliable extrapolation.

The claim that a bridgehead alkene evades large twisting angles $\theta$ by pyramidalization at the methine carbon is based primarily on theory but is supported by the values found for the methine $\mathrm{C}-\mathrm{H}$ stretching and bending frequencies of homoadamantene (13) and adamantene (15) as discussed earlier. ${ }^{1}$ The methine pyramidalization degree of freedom makes it clearly impossible to attain values of the twist angle $\theta$ anywhere near $90^{\circ}$ in a bridgehead alkene with a trisubstituted double bond, and more elaborate polycyclic structures or perhaps a very bulky fourth substituent are necessary to enforce a nearly orthogonal twist. Even in adamantene, whose heavy-atom skeleton has a twist angle of about $80^{\circ}$, the value of $\theta$ is only $64^{\circ}$ and we suspect that $\theta$ will not exceed $70^{\circ}$ in any trisubstituted bridgehead double bond. This corresponds to still quite considerable $\pi$ bond strength and there is no doubt that even the most highly twisted bridgehead alkenes will exist as a pair of geometrical isomers or enantiomers separated by a considerable barrier.

Too few data points are presently available for any generalizations concerning the singlet $\pi^{*}$ excitation energies of trisubstituted bridgehead double bonds (Table 2), but for the above reasons it is not likely that they will drop below $25000 \mathrm{~cm}^{-1}$ even in the most highly twisted representatives of this class of compounds.

\section{CONCLUSIONS}

Although a fair amount of progress has been made in establishing the structures and spectral properties of bridgehead imines and, to a lesser degree, alkenes, much remains to be done before the fundamental questions concerning their molecular and electronic structure are satisfactorily answered, and investigations of their reactivity have only barely begun. 
For imines, there now is a quite safe correlation between the cosine of the twist angle $\theta$ on the one hand and either the $C=N$ stretching frequency or the $n \pi^{*}$ or $\pi^{*}$ excitation energy on the other, the limits for orthogonal twist (and otherwise the usual valence angles) being $1350-1400 \mathrm{~cm}^{-1}$ for the $\mathrm{C}=\mathrm{N}$ stretch and the edge of the IR region for the $n \pi^{*}$ excitation. The data obtained permit good qualitative insight into the mechanism of photochemical syn-anti isomerization.

For bridgehead alkenes, the existence of similar correlations can be suspected but has not been established and additional data points are needed before extrapolation to the orthogonal twist limit can be attempted. It appears that the highest twist angles $\theta$ that can be forced on a trisubstituted bridgehead alkene are only of the order of $70^{\circ}$ and more complex polycyclic structures will need to be devised, or more highly substituted derivatives used, to arrive at twist angles near orthogonality.

Acknowledgement. This work was supported by U.S. National Science Foundation (CHE 8421117).

\section{REFERENCES}

1. J. Michl, J. G. Radziszewski, J. W. Downing, K. B. Wiberg, F. H. Walker, R. D. Miller, P. Kovacic, M. Jawdosiuk, and V. Bonalic-Koutecky, Pure Appl. Chem., 55, 315 (1983).

2. V. BonaliC-Koutecky and J. Michl, Angew. Chem. Internat. Ed. Engl., in press (1986).

3. J. Michl, Photochem. Photobiol., 25, 141 (1977).

4. R. C. Haddon, J. Am. Chem. Soc., 108, 2837 (1986).

5. N. L. Allinger and J. T. Sprague, J. Am. Chem. Soc., 94, 5734 (1972).

6. J. G. Radziszewski, T.-K. Yin, F. Miyake, G. E. Renzone, W. T. Borden, and J. Michl, J. Am. Chem. Soc., 108, 3544 (1986).

7. J. G. Radziszewski, J. W. Downing, M. Jawdosiuk, P. Kovacic, and J. Michl, J. Am. Chem. Soc., 107, 594 (1985); see also J. G. Radziszewski, Ph.D. dissertation, University of Marburg, Germany, 1985.

8. V. BonaClic-Kouteckg and J. Michl, Theor. Chim. Acta, 68, 45 (1985).

9. J. G. Radziszewski, J. W. Downing, C. Wentrup, P. Kaszynski, M. Jawdosiuk, P. Kovacic, and J. Michl, J. Am. Chem. Soc., 106, 7996 (1984).

10. J. G. Radziszewski, J. W. Downing, C. Wentrup, P. Kaszynski, M. Jawdosiuk, P. Kovacic, and J. Mich1, J. Am. Chem. Soc., 107, 2799 (1985).

11. R. T. Conlin, R. D. Miller, and J. Michl, J. Am. Chem. Soc., 101, 7637 (1979).

12. D. J. Martella, M. Jones, Jr., P. v.R. Schleyer, and W. F. Maier, J. Am. Chem. Soc., 101, 7634 (1979). 